\title{
Set theoretic estimation in color scanner characterization
}

\author{
Gaurav Sharma* \\ H. Joel Trussell \\ North Carolina State University \\ Electrical and Computer Engineering Department \\ Raleigh, North Carolina 27695-7911 \\ E-mail: hjt@eos.ncsu.edu
}

\begin{abstract}
A set theoretic approach for the spectral characterization of a color scanner is described. These devices usually employ three channels to obtain a device dependent RGB (red, green, blue) image. To display/print an image, the device dependent $R G B$ values must be correctly transformed to the color space of the target device. To determine accurate and efficient transformations for a number of devices, knowledge of the spectral sensitivity of the scanner is essential. Direct measurement of the sensitivity requires a set of expensive narrow band reflectances and is often infeasible. Methods that estimate the spectral sensitivity based on measurements with typical reflectance samples are therefore of interest. Due to the low dimensionality of the space of object reflectance spectra, this is a highly ill-conditioned problem. As a result, conventional estimation techniques that fail to take a priori knowledge into account perform rather poorly on this problem. A set theoretic approach that incorporates available a priori knowledge into the estimation framework yields better results. Results are presented for a simulated scanner characterization problem and for an actual characterization to demonstrate the increased accuracy compared with conventional methods. (0) 1996 SPIE and IS\&T.
\end{abstract}

\section{Introduction}

With increasing exchange of image data between different platforms, there has been a rising interest in transformations that translate color data obtained on one device into the native color space of another device with minimal degradation in color. ${ }^{\dagger}$ To this end, attempts are underway to standardize the mechanisms for specifying these transformations and for their inclusion in standard image formats as device profiles. ${ }^{2}$ The creation of profiles for color scanners requires a characterization of these devices. Calibration schemes that attempt to transform the scanner data into a standardized device independent color space have been studied extensively. ${ }^{3-6}$ However, the more fundamental problem of estimating the spectral sensitivity of the scanner *Current affiliation: Digital Imaging Technology Center, Xerox Corp., 800 Phillips
Rd., Webster, NY 14580. E-mail: sharma@wrc.xerox.com.

${ }^{\dagger}$ For the purposes of this paper the knowledge of color standards and terminology is not required. The interested reader can, however, refer to Ref. 1 for a tutorial introduction to color signal processing.

Paper 95-039 received Dec. 4, 1995; revised manuscript received May 3, 1996; accepted for publication May 9, 1996.

1017-9909/96/\$6.00 @ 1996 SPIE and IS\&T. has received only limited attention. ${ }^{7-10}$ The spectral characterization can be used to determine the optimal transformation of the color data into the output device space ${ }^{11,12}$ instead of the suboptimal two-step procedure involving the transformation to an intermediate device independent color space. The spectral characterization can also be used for estimating dye sensitivities from scans of photographic prints $^{13}$ and in more general color separation applications aimed at high quality color hardcopy. It is also of independent interest in gauging the colorimetric quality of the scanner ${ }^{14}$ at the user's end and can form the basis of hardware improvements in the scanner.

\section{Scanner Model}

The output of a color scanner is a three-band image, typically in red, green and blue channels. Assuming that the scanner sensors are linear and that the spatial response of the scanner is the ideal 2-D impulse function, ${ }^{*}$ the scanning process can be modeled as

$$
\begin{aligned}
t^{(i)} & =\int_{-\infty}^{\infty} f_{i}(\lambda) \theta(\lambda) r(\lambda) l(\lambda) d \lambda+\epsilon^{(i)} \\
& =\int_{-\infty}^{\infty} m_{i}(\lambda) r(\lambda) l(\lambda) d \lambda+\epsilon^{(i)}, \quad i=1,2,3
\end{aligned}
$$

where $f_{1}(\lambda), f_{2}(\lambda)$, and $f_{3}(\lambda)$ are the spectral transmittances of the three color filters; $\theta(\lambda)$ is the sensitivity of the detector used in the measurements; $l(\lambda)$ is the spectral radiance of the illuminant; $r(\lambda)$ is the spectral reflectance of the area being scanned; $\boldsymbol{\epsilon}^{(i)}$ is the measurement noise, $m_{i}(\lambda)=f_{i}(\lambda) \theta(\lambda)$ is the product of filter transmittance and detector sensitivity, and $t^{(i)}$ denotes the value obtained from the $i$ 'th channel.

\footnotetext{
The problem of determining the spatial response can be separated from that of estimating the spectral sensitivity by using reasonable large color patches for the spectral characterization.
} 
If the spectra are all band-limited, they can be represented in terms of their samples, and the integral can then be approximated by a summation. If samples at $N$ equispaced wavelengths are used, the scanning process can be represented algebraically as

$t^{(i)}=\mathbf{r}^{T} \mathbf{L} \mathbf{m}_{i}+\boldsymbol{\epsilon}^{(i)}, \quad i=1,2,3$,

where $\mathbf{r}$ is the $N \times 1$ vector of reflectance samples, $\mathbf{L}$ is an $N \times N$ diagonal matrix with samples of the radiant spectrum of the illuminant along the diagonal, and $\mathbf{m}_{i}$ is an $N \times 1$ vector of samples of the product of the $i$ 'th filter transmittance and the detector sensitivity.

Reflectances encountered in scanned images are typically smooth functions ${ }^{15-17}$ of wavelength and can be sampled with negligible aliasing using a 10-nm sampling interval. The same is true of transmittances of typical filters used in color scanners, detector sensitivities and incandescent lamp spectra. However, a $10-\mathrm{nm}$ sampling interval is inadequate for fluorescent lamps which have sharp emission lines in their spectra. ${ }^{18,19}$ A much smaller sampling interval could be used in Eq. (2), but this leads to a large increase in the dimensionality of the problem with the attendant disadvantages of larger computational load and greater ill-conditioning. For instance, if the visible region from 380 to $780 \mathrm{~nm}$ is used, a 10-nm sampling interval leads to $N=41$ samples whereas a 2 -nm sampling (required for the accurate representation of a fluorescent lamp) would raise the dimensionality of the problem to $N=201$.

Fluorescent lamps are often used in desktop scanners on account of their higher efficiency and lower heat dissipation compared with incandescent lamps. Hence, the problem of increased dimensionality (due to a high sampling rate) is frequently encountered in practice. As a remedy for the high sampling rate, it is possible to use a decomposition for fluorescent illuminant spectrum in terms of the smooth spectrum emitted by the phosphor coating on the lamp envelope, and monochromatic spectral peaks corresponding to the emission lines of the gaseous vapor in the lamp. ${ }^{19-21}$ Appendix A describes the mechanism for incorporating the illuminant decomposition into the scanner model. The resulting scanner model in Eq. (26) of Appendix A has the same form as Eq. (2) except that the illuminant matrix $\mathbf{L}$ is replaced by the effective illuminant matrix $\mathbf{L}_{e}$, which is not necessarily diagonal. Therefore, in the subsequent discussion, Eq. (2) is used with the understanding that if the illuminant is a fluorescent lamp, either a high sampling rate is used ( $2 \mathrm{~nm}$ in our discussion, which yields $N=201$ ) or $\mathbf{L}_{e}$ replaces $\mathbf{L}$.

The spectral response of the scanner is given by the illuminant sensitivity products $\left\{\mathbf{L m}_{i}\right\}_{i=1}^{3}$ for the three channels. For initial calibration, the individual quantities can be measured before assembly, and the scanner sensitivity computed from those. However, such a calibration would deteriorate with time due to the aging of the components. ${ }^{22}$ In addition, this calibration would represent a significant expense for these low cost devices and is rarely done. For an in situ characterization of the scanner, one has to estimate the sensitivities by using the scanner measurements for a target comprised of samples with known reflectance spectra, $\left\{\mathbf{r}_{k}\right\}_{k=1}^{K}$,
$t_{k}^{(i)}=\mathbf{r}_{k}^{T} \mathbf{L} \mathbf{m}_{i}+\boldsymbol{\epsilon}^{(i)}, \quad i=1,2,3$.

This set of equations can be written as,

$\mathbf{t}^{(i)}=\mathbf{R}^{T} \mathbf{L} \mathbf{m}_{i}+\boldsymbol{\epsilon}^{(i)}$,

where $\mathbf{t}_{K \times 1}^{(i)}=\left[t_{1}^{(i)}, t_{2}^{(i)} \ldots t_{K}^{(i)}\right]^{T}$ is the vector of measurements from the $i$ 'th channel, and $\mathbf{R}=\left[\mathbf{r}_{1}, \mathbf{r}_{2} \ldots \mathbf{r}_{K}\right]$ is the matrix of reflectance spectra used in the measurements.

The problem of obtaining spectral characterization of the scanner is now one of estimating $\left\{\mathbf{L m}_{i}\right\}_{i=1}^{3}$ from the measurement vectors $\left\{\mathbf{t}^{(1)}, \mathbf{t}^{(2)}, \mathbf{t}^{(3)}\right\}$. It is shown in Section 5 that an independent and direct measurement of the illuminant improves the final estimates of the sensitivity considerably. This can often be accomplished without much difficulty. For fluorescent lamps, a technique for obtaining the decomposition of Eq. (23) of Appendix A from measurements made with band-limited sensors has been demonstrated in Ref. 20.

The sensitivity estimation is considered for two independent cases: the first in which the illuminant spectrum is measured and known a priori so that $\left\{\mathbf{m}_{i}\right\}_{i=1}^{3}$ needs to be estimated, and the second in which there is no a priori knowledge about the illuminant and the illuminant sensitivity products $\left\{\mathbf{L} \mathbf{m}_{i}\right\}_{i=1}^{3}$ are to be estimated. For a unified treatment of both cases, it is convenient to write Eq. (4) in the form

$\mathbf{t}^{(i)}=\mathbf{W}^{T} \mathbf{s}_{i}+\boldsymbol{\epsilon}^{(i)}, \quad i=1,2,3$,

where $\mathbf{s}_{i}=\mathbf{L} \mathbf{m}_{i}, \mathbf{W}=\mathbf{R}$ if the illuminant spectrum is not known a priori and $\mathbf{s}_{i}=\mathbf{m}_{i}$, and $\mathbf{W}=\mathbf{L}^{T} \mathbf{R}$ if the illuminant spectrum is known a priori. Thus in either case, $\mathbf{W}$ represents the known information and $\left\{\mathbf{s}_{i}\right\}_{i=1}^{3}$ represent the unknown quantities to be estimated. Note here that the same framework can be used when the scanner has a fluorescent lamp and knowledge of the illuminant is limited to the location of the spectral peaks. Appendix B provides the details for this case.

The vectors $\left\{\mathbf{s}_{1}, \mathbf{s}_{2}, \mathbf{s}_{3}\right\}$ need to be estimated for characterizing the scanner. Since the correlation between the different channel sensitivities is not known, the only techniques considered here will be those which use only the vector $\mathbf{t}^{(i)}$ to estimate $\mathbf{s}_{i}$. It will therefore be notationally efficient to consider the estimation of the sensitivity $\mathbf{s}_{N \times 1}$ given the measurements

$\mathbf{t}_{K \times 1}=\mathbf{W}^{T} \mathbf{s}+\boldsymbol{\epsilon}$,

with the understanding that the estimation procedure is performed independently for the three channels.

\section{Least Squares and Principal Eigenvector Solutions}

A simple procedure to estimate the scanner sensitivity would be to solve the least-squares problem

$\hat{\mathbf{s}}=\arg \min \left\|\mathbf{t}-\mathbf{W}^{T} \mathbf{y}\right\|$, 
where the minimization is performed over all possible $N \times 1$ vectors, and $\mathbf{W}$ is computed from $\mathbf{R}$ as indicated earlier.

The solution to this problem is readily obtained in terms of the Moore-Penrose pseudoinverse. ${ }^{23,24}$ In terms of the singular value decomposition ${ }^{23}$ (SVD), $\mathbf{W}=\mathbf{U} \boldsymbol{\Lambda} \mathbf{V}^{\mathbf{T}}$, the least-squares solution can be written as

$\hat{\mathbf{s}}=\sum_{i=1}^{P} \frac{\left(\mathbf{v}_{i}^{T} \mathbf{t}\right)}{\sigma_{i}} \mathbf{u}_{i}$,

where $P[\leqslant \min (K, N)]$ is the rank of $\mathbf{W} ;\left\{\sigma_{i}\right\}_{i=1}^{P}$ are the nonzero singular values of $\mathbf{W}$ (in decreasing order); and $\left\{\mathbf{u}_{i}\right\}_{i=1}^{N}$ and $\left\{\mathbf{v}_{i}\right\}_{i=1}^{N}$ are the columns of $\mathbf{U}$ and $\mathbf{V}$, respectively (the left and right singular vectors of $\mathbf{W}$ ).

The least-squares approach suffers from a serious practical problem in that the spectra of natural objects do not have sufficient dimensionality to yield a good estimate of s. If the noise is assumed to be white, the mean squared estimation error for least squares is given by,

$E\left\{\|\mathbf{s}-\hat{\mathbf{s}}\|^{2}\right\}=\sum_{i=P+1}^{N}\left(\mathbf{u}_{i}^{T} \mathbf{s}\right)^{2}+\sum_{i=1}^{P} \frac{\sigma_{\epsilon}^{2}}{\sigma_{i}^{2}}$,

where $E$ denotes the expectation operator, and $\sigma_{\epsilon}^{2}$ is the noise variance. Typically, the matrix of reflectance spectra $\mathbf{R}$ is highly ill-conditioned and has only seven to eight significant singular values. ${ }^{15,25}$ As a result, even at low levels of noise the higher order singular values of $\mathbf{W}$ are small compared with $\sigma_{\epsilon}^{2}$, and the second summation in Eq. (9) is large. Therefore, the least-squares solution is highly sensitive to noise and yields extremely poor estimates of the spectral sensitivity at noise levels typical in desktop scanners.

The sensitivity of the least-squares solution to noise is greatly reduced if only the singular vectors corresponding to the significant singular values are used in the solution, i.e., if the singular vectors $\mathbf{v}_{i}$ for which $\sigma_{i}$ is small are not included in the solution. If the singular values beyond $\sigma_{P^{\prime}}$ are considered insignificant, the estimate of the sensitivity becomes

$\hat{\mathbf{s}}=\sum_{i=1}^{P^{\prime}} \frac{\left(\mathbf{v}_{i}^{T} \mathbf{t}\right)}{\sigma_{i}} \mathbf{u}_{i}$

This solution, referred to as the principal eigenvector (PE) solution, $8,24,26,27$ is far less sensitive to noise than the leastsquares solution and is tested in Section 5.

\section{Method of Projections onto Convex Sets}

While the PE method is less sensitive to noise than the least-squares solution, it still fails to take into account the considerable a priori knowledge available from the physical situation. For instance, except for the illuminant, the scanner sensitivity is a smooth function of wavelength. Hence if the illuminant is known, the functions $\left\{\mathbf{s}_{j}=\mathbf{m}_{j}\right\}_{j=1}^{3}$ are smooth. However, if the illuminant has sharp peaks in its spectrum, the principal eigenvectors of
$\mathbf{W}=\mathbf{L R}$ will also have sharp peaks and will yield estimates of $\left\{\mathbf{s}_{j}\right\}_{j=1}^{3}$ that have sharp spectral peaks. On the other hand, if the illuminant is a fluorescent lamp whose spectrum is not known, the functions $\left\{\mathbf{s}_{j}=\mathbf{L} \mathbf{m}_{j}\right\}_{j=1}^{3}$ have sharp spectral peaks, but for typical reflectance samples the principal eigenvectors of $\mathbf{W}=\mathbf{R}$ will be smooth and therefore yield only smooth estimates of $\left\{\mathbf{s}_{j}\right\}_{j=1}^{3}$. In addition to this knowledge of smoothness/impulsiveness, other a priori information such as non-negativity of the sensitivity functions and boundedness can be incorporated in a set theoretic estimate. ${ }^{28}$ Based on each constraint that the scanner sensitivity must satisfy, a set can be defined in which the true value of the sensitivity must lie. Any element in the intersection of the constraint sets is then a feasible solution and can be used as an estimate of the sensitivity. If only closed convex constraint sets are used, a point in the intersection can be determined by projecting onto the sets in cyclic order starting from an arbitrary point in $\mathbb{R}^{N}$. This is the well-known method of successive projections onto convex sets $^{28,29}$ (POCS). If one has $n$ constraint sets, the POCS estimate is the limit of the sequence $\left\{\mathbf{y}_{k}\right\}$ defined recursively by,

$\mathbf{y}_{k+1}=P_{n}\left(P_{n-1}\left(\ldots P_{2}\left(P_{1}\left(\mathbf{y}_{k}\right)\right) \ldots\right)\right)$,

where $\mathbf{y}_{0}$ is an arbitrary starting point, and $P_{i}(\mathbf{z})$ denotes the projection of $\mathbf{z}$ onto the $i$ 'th constraint set, defined as the vector in the $i$ 'th constraint set closest to $\mathbf{z}$. The iterative process of successive projections is guaranteed to converge to a point in the intersection, provided the intersection is nonempty. ${ }^{29,30}$ The same convergence result holds for several variants of the basic method that use relaxation/ parallelization to speed up convergence. ${ }^{31}$

Based on the physical nature of the problem and the measurement model, it can be said that the following constraint sets can be used for the sensitivity function $\mathbf{s}$ :

1. The set of non-negative vectors

$$
A_{n}=\left\{\mathbf{y} \in \mathbb{R}^{N} \mid y_{i} \geqslant 0, \quad \forall 1 \leqslant i \leqslant N\right\} .
$$

2. The noise variance set

$$
A_{\epsilon}=\left\{\mathbf{y} \in \mathbb{R}^{N} \mid\left\|\mathbf{t}-\mathbf{W}^{T} \mathbf{y}\right\|^{2} \leqslant \nu\right\},
$$

where the upper bound $\nu$ is determined from the statistics of the noise to ensure a reasonable confidence level.* If the noise is assumed Gaussian, $\left\|\mathbf{t}-\mathbf{W}^{T} \mathbf{y}\right\|^{2}$ is a chi-squared random variable with $K$ degrees of freedom. Usually, $K$ is large enough for the central limit theorem to apply and in that case, the $95 \%$ confidence level is attained for ${ }^{32,33}$

$$
\nu=K \sigma_{\epsilon}^{2}\left(1+\lim _{0.95} \sqrt{\frac{2}{K}}\right),
$$

\footnotetext{
* A confidence interval would typically involve both a lower and an upper bound, but in practice the other constraints ensure that the residual energy lies above the lower bound.
} 
where $\lim _{0.95}$ is the $95 \%$ confidence limit for the standard normal distribution, and $\sigma_{\epsilon}^{2}$ is the noise variance.

3. The noise outlier sets

$A_{o}^{i}=\left\{\mathbf{y} \in \mathbb{R}^{N}|| \mathbf{t}_{i}-\mathbf{w}_{i}^{T} \mathbf{y} \mid \leqslant \xi\right\} \quad i=1,2, \ldots K$,

where $\mathbf{w}_{i}$ is the $i$ th column of $\mathbf{W}$ and $\xi \approx 3 \sigma_{\epsilon}$ ensures a $99 \%$ confidence level for Gaussian noise.

4. The passive response set

$A_{p}=\left\{\mathbf{y} \in \mathbb{R}^{N} \mid y_{i} \leqslant 1, \quad \forall 1 \leqslant i \leqslant N\right\}$

can be used if the illuminant is known a priori, and the combined effect of the passive filter and detector responses is to be determined. Even if the illuminant is unknown or known only up to a constant scale factor, the physical situation typically provides us an upper bound of the form in Eq. (16). This bound can still be used though it may not be too tight. More useful bounds can be established in terms of possible deviations from a measured prototype. ${ }^{34}$ These would vary over the wavelength range unlike the uniform bound of Eq. (16).

5. A smoothness constraint set can be used when the sensitivities $\{\mathbf{s}\}_{j=1}^{3}$ are known to be smooth functions of wavelength. The set of smooth spectra can be defined in terms of an upper bound on the energy in a high-pass filtered output

$A_{s}=\left\{\mathbf{y} \in \mathbb{R}^{N} \mid\|\mathbf{H} \mathbf{y}\|^{2} \leqslant \mu\right\}$,

where $\mathbf{H}$ represents the convolution operator for the high-pass filter with impulse response $\mathbf{h}^{27}$ and $\mu \geqslant 0$ is chosen so as to impose the desired degree of smoothness. The filter $\mathbf{h}$ can be chosen in a number of different ways depending on the nature of the knowledge that is available regarding the smoothness. If an absolute bandlimit is known, a perfect high-pass filter can be chosen with $\mu=0$. Alternately, if it is desirable to allow some energy in the highpass region, filters that approximate derivatives of different orders can be used. ${ }^{35}$ Prototypes of the sensitivity functions can then be used to estimate the upper bound $\mu . .^{34,35}$ The Laplacian filter, h $=(1,-2,1)^{T}$, which approximates the second derivative operation is one possible choice for the filter in the smoothness constraint.

6. A concave or unimodal set can be used when the color filters used are known to be unimodal. The set of unimodal filters does not constitute a convex set and therefore cannot be used in POCS. However, unimodality can be enforced in practice by requiring the estimated vector to be concave over a certain wavelength range. In terms of the samples of the scanner sensitivity, this restriction can be imposed by requiring that the Laplacian approximation to the second derivative be nonpositive over a specified set of indices. The corresponding sets can be defined as,

$A_{c}^{i}=\left\{\mathbf{y} \in \mathbb{R}^{N} \mid[\mathbf{H y}]_{i} \leqslant 0\right\} \quad i \in \mathscr{T}$, where $\mathbf{H}$ denotes the Laplacian filter, $[\mathbf{z}]_{i}$ denotes the $i$ 'th component of the vector $\mathbf{z}$, and $\mathscr{T}$ is the set of indices that defines the wavelength range over which the sensitivity is known to be concave.

7. The set of vectors close to the human visual space (HVS) can be used if it is known that the scanner sensitivities were designed to approximate color mixture curves, i.e., close to linear transformations of the CIE color matching functions. ${ }^{36}$ The corresponding set can be defined as,

$A_{m}=\left\{\mathbf{y} \in \mathbb{R}^{N} \mid\left\|\left(\mathbf{I}-\mathbf{P}_{v}\right) \mathbf{y}\right\|^{2} \leqslant \kappa\right\}$,

where $\mathbf{P}_{v}$ denotes the orthogonal projection matrix that projects onto the HVS $\mathrm{H}^{14,37}$ and the upper bound $\kappa$ is determined by the closeness of the sensitivity to the HVS.

It can be readily verified that each of the constraint sets just defined is convex and closed. The projection operators for these constraint sets can be calculated using standard nonlinear programming techniques ${ }^{38}$ and are tabulated in Appendix C.

The POCS estimate is typically nonunique and depends on the initial point $\mathbf{y}_{0}$ chosen to start the iterations. Typically the converged solution is close to the initial point chosen to commence the iterations. ${ }^{39}$ The global convergence guaranteed by POCS can be used to incorporate imprecise a priori information into the estimation through a proper choice of the initial estimate. The estimate to which POCS converges may be appropriately perturbed to incorporate information not captured in the POCS framework, and the algorithm repeated to obtain estimates that agree better with the new information. A "more" unimodal estimate can be obtained, for example, by repeating POCS commencing with the concave hull of the original estimate.

\section{Simulation Results}

To compare the performance of the PE and the POCS estimators, simulations were conducted using the scanner model of Eq. (2). The WR-25 red, WR-40 green, and WR38a blue filters from the "Wratten" filter set were chosen as the color filters. The detector response was assumed to be uniform and UV (GG400) and IR (KG4) cutoff filters from Schott's catalog of commercial glass filters ${ }^{40}$ were used to ensure that the response is restricted to the visible region of the spectrum. The spectrum of a fluorescent lamp from an existing desktop scanner was used as the illuminant. The illuminant spectrum is shown in Fig. 1.

For comparison of the different algorithms, the Kodak Q60 target ${ }^{41}$ and the Munsell chart ${ }^{15}$ were used. The reflectance spectra for the 240 blocks in the Kodak Q60 target and the 64 Munsell chips were measured independently using a spectrophotometer. A randomly chosen subset containing half of these, i.e., $K=152$ spectra, was used as the calibration target. The remaining 152 reflectances were used as a test target. Note that the nature of the results of this section does not change appreciably if a different or somewhat smaller set of reflectances is used. 


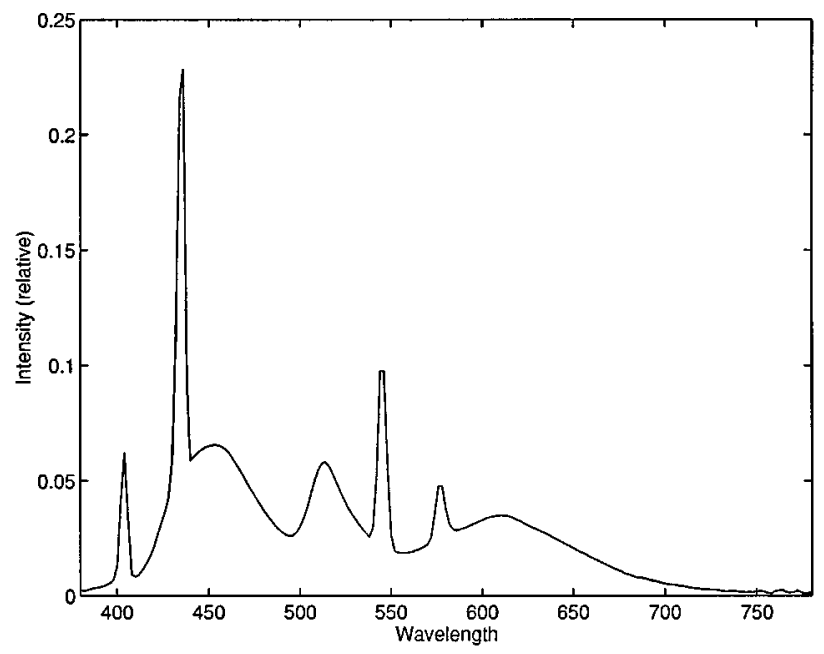

Fig. 1 Spectrum of illuminant used in simulations.

Simulated noisy measurements of scanner RGB values were made using the calibration target reflectances in the model of Eq. (2), where the noise $\epsilon_{i}$ was generated using a Gaussian random number generator. The value of the noise variance $\sigma_{\epsilon}^{2}$ was calculated assuming a signal-to-noise ratio* (SNR) of $33 \mathrm{~dB}$ in conjunction with the definition

$\operatorname{SNR}(\mathrm{dB})=10 \log _{10}\left(\frac{\left\|\mathbf{W}^{T} \mathbf{s}\right\|^{2}}{K \sigma_{\epsilon}^{2}}\right)$.

In the principal eigenvector method, $P^{\prime}=8$ was used for this set of reflectances. ${ }^{\dagger}$

Two sets of simulations were conducted. In the first experiment no prior knowledge of the illuminant was assumed. In the notation of Section 2, this corresponds to the case where the estimated sensitivity is the product of the illuminant and the optical path transmittance, $\mathbf{s}_{i}=\mathbf{L m}_{i}$, and $\mathbf{W}=\mathbf{R}$. Since the illuminant is a fluorescent lamp with sharp emission lines in its radiant spectrum, the scanner sensitivity will also have sharp spectral peaks and therefore a 2-nm sampling interval is necessary for the simulation. This yields $N=201$ samples in the visible region from 380 to $780 \mathrm{~nm}$. The noisy "measurements" and the known matrix of target reflectance spectra were used in the PE and the POCS methods to obtain estimates of the scanner sensitivity. Since in this case the sensitivity (which includes the fluorescent illuminant) is neither smooth nor concave over a spectral region of appreciable spread, the

\footnotetext{
*The SNR was arrived at by actually estimating the noise level in a commercial 8 bit/channel flatbed color scanner.

The nature of the results does not change if any integer between 6 and 10 is used instead of 8 .
}

While, it is known that the sensitivity has sharp peaks this information cannot be readily incorporated in the POCS estimation scheme on account of its nonconvex nature.

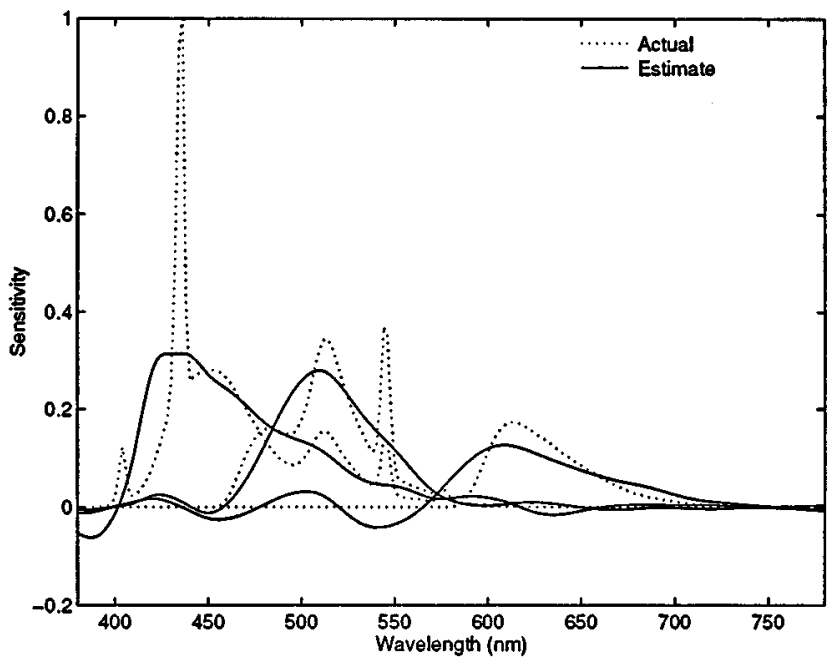

Fig. 2 PE estimate; illuminant unknown case.

smoothness and concavity constraint sets were not used for POCS. ${ }^{\ddagger}$ The estimates for the scanner sensitivity for the three channels are plotted in Fig. 2 for the PE method and in Fig. 3 for the POCS method. For comparison both figures include plots of the actual sensitivities used in generating the simulated measurements. The actual sensitivities are nonnegative functions with sharp spectral peaks. The PE estimates are smooth and have negative lobes that are physically impossible. The POCS estimates are also smooth, but do not have any negative lobes due to the imposed nonnegativity constraint. The a priori information in this case is rather limited, and hence POCS does not perform significantly better than the PE method.

In the second simulation, the illuminant spectrum was assumed to be known a priori, so that only the optical path transmittances needed to be estimated. In the notation of Section 2, this corresponds to the case with $\mathbf{s}_{i}=\mathbf{m}_{i}$ and $\mathbf{W}=\mathbf{L R}$. For this case since the sensitivity is smooth and unimodal, both the smoothness and concavity constraints were used in POCS.

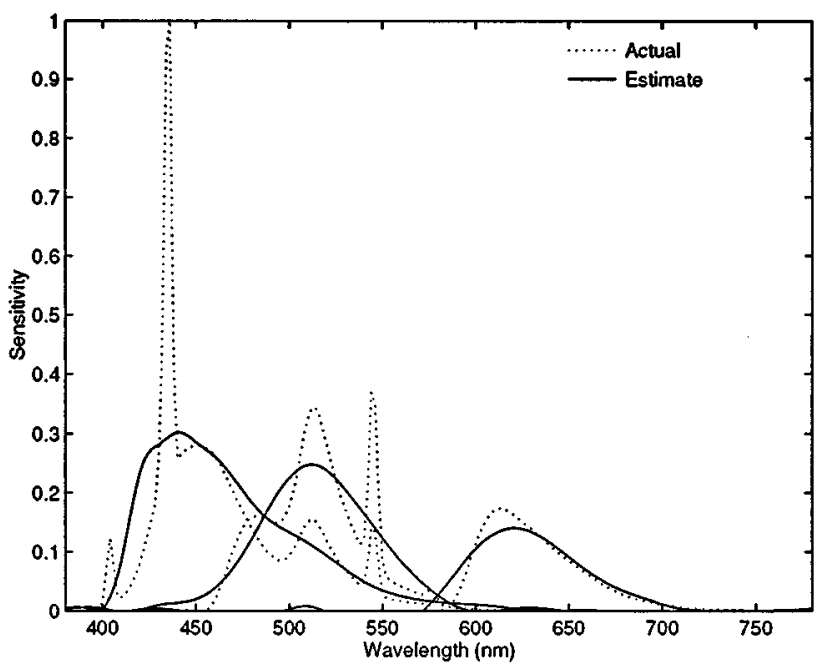

Fig. 3 POCS estimate; illuminant unknown case. 


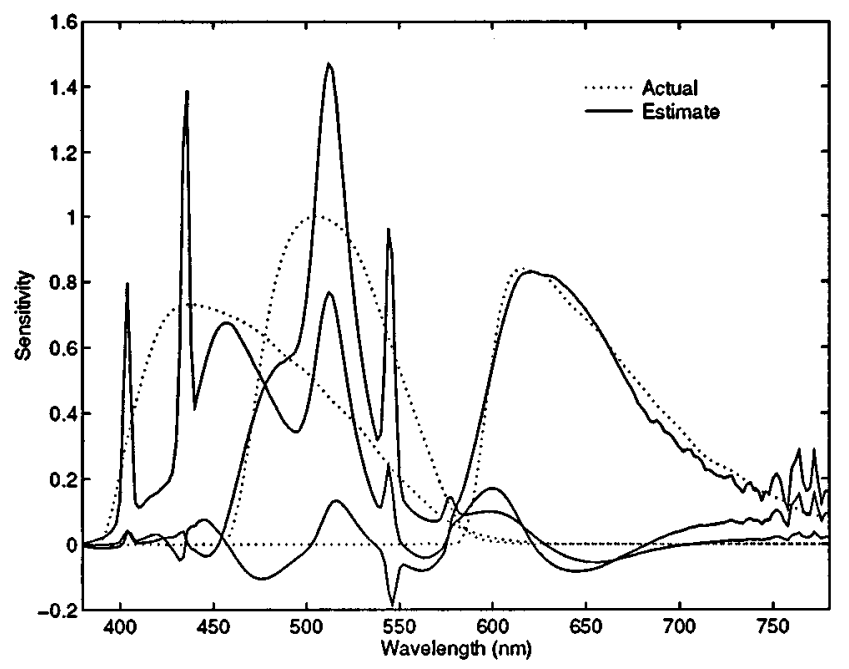

Fig. 4 PE estimate; illuminant known case, 2-nm sampling.

Two further subcases were considered in this simulation to test the effect of the illuminant model of Eq. (23). In the first the sampling rate of $2 \mathrm{~nm}$ necessary for accurate sampling of the illuminant was used for the reflectance spectra and for the sensitivity, and therefore the dimensionality of the problem remained $N=201$. In the second subcase, the illuminant model described in Eqs. (24) to (27) was used with a 10-nm sampling for the reflectance spectra and the sensitivity, reducing the dimensionality of the problem to $N=41$. The estimates obtained for the sensitivity functions of the three color channels with the 2-nm sampling are shown in Fig. 4 for the PE method and in Fig. 5 for POCS. The corresponding results for the characterization using the illuminant model and 10-nm sampling are shown in Figs. 6 and 7, respectively. Note that the POCS estimates for the two different sampling rates are quite close and therefore validate the model. The use of the model instead of the higher sampling rate greatly reduces the dimensionality of

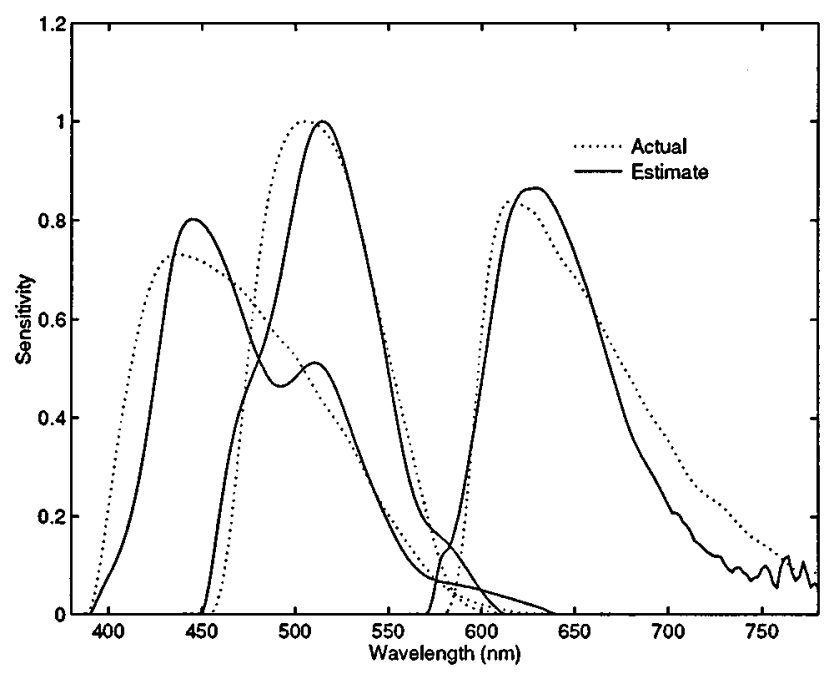

Fig. 5 POCS estimate; illuminant known case, 2-nm sampling.

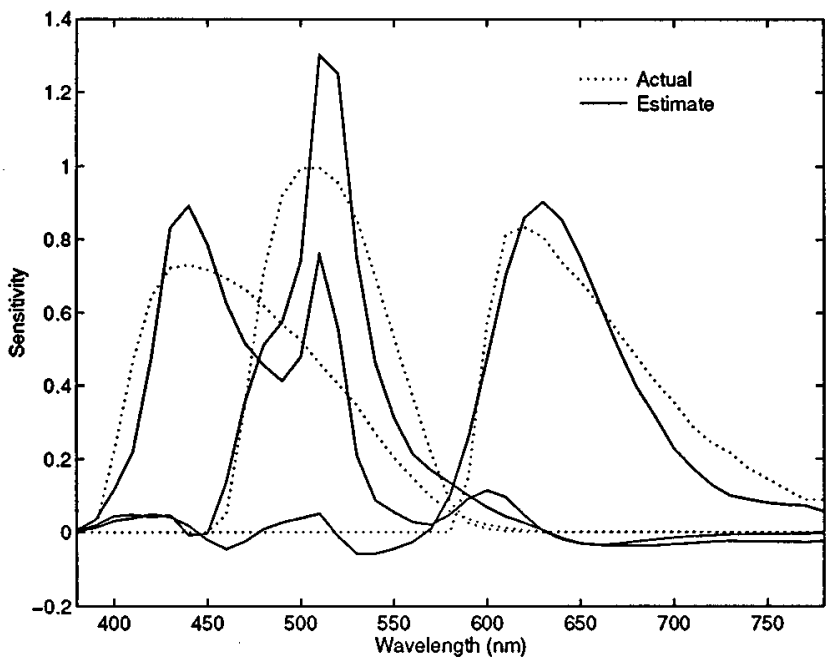

Fig. 6 PE estimate; illuminant known case, 10-nm sampling with illuminant model.

the problem and leads to faster convergence (for POCS) and, as shown later, more accurate estimates.

The PE and POCS estimates exhibit interesting differences. Since the illuminant has sharp peaks in its spectrum, the left singular vectors of $\mathbf{W}(=\mathbf{L R})$ are not smooth. This remains true even when the sampling rate of $10 \mathrm{~nm}$ is used in conjunction with the illuminant model. Therefore, as noted in Section 4, the estimates of $\left\{\mathbf{s}_{j=1}^{3}\right.$ obtained by the PE method [see Eq. (10)] also exhibit sharp peaks. Additionally, in both cases the PE estimates of the sensitivity have large negative regions that are inconsistent with the physical nature of the sensitivity. The POCS estimates agree with all the constraints imposed and are therefore nonnegative, smooth functions with concave regions around the peaks. The difference in the estimates indicates the utility of a priori information for the characterization.

To quantify the accuracy of the estimates, two different error metrics were considered: the mean squared estimation

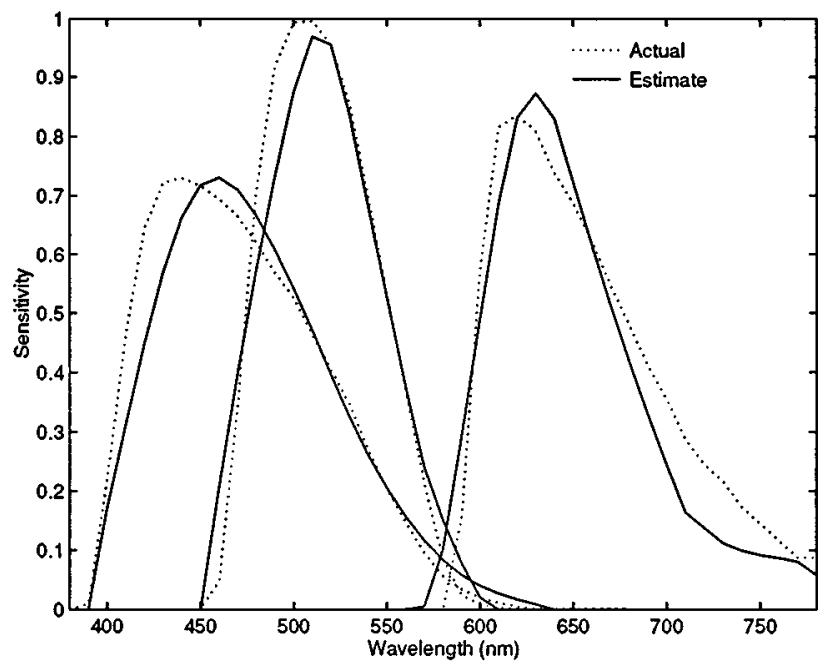

Fig. 7 POCS estimate; illuminant known case, 10-nm sampling with illuminant model. 
Table 1 Mean squared estimation error of spectra for $P E$ and POCS estimates.

\begin{tabular}{lcrrr}
\hline \hline \multirow{2}{*}{ Estimation Scheme } & & \multicolumn{3}{c}{ Mean Squared Error (dB) } \\
\cline { 3 - 5 } & & Red & Green & Blue \\
\hline \multirow{2}{*}{ Illuminant unknown } & PE 2 nm & -6.88 & -9.54 & -5.01 \\
& POCS 2 nm & -13.56 & -9.74 & -5.28 \\
Illuminant known & PE 2 nm & -14.64 & -8.45 & -6.23 \\
& POCS 2 nm & -15.11 & -15.19 & -12.94 \\
& PE 10 nm & -15.74 & -10.39 & -12.01 \\
& POCS 10 nm & -15.47 & -17.62 & -16.74 \\
\hline \hline
\end{tabular}

error and the error in predicted scanner RGB values. For both estimation schemes, the mean squared estimation error was computed (for each of the three channels) as the average squared difference between the actual value and the estimate of the sensitivity. The resulting numerical values are presented in Table 1. From the tabulation one can see that the performance of the PE and POCS methods is similar when the illuminant spectrum is not known and there is limited prior knowledge (first two rows of Table 1). However, when the illuminant spectrum is known a priori, the performance of POCS improves radically while that of the PE method does not. For the two subcases for the known illuminant case, POCS is significantly better than PE for the 2-nm sampling and much better for the 10-nm sampling with the illuminant model.

For the computation of mean squared error in scanner RGB values, the test target was used in the model of Eq. (2) with the noise term set to zero. Recall that the test target was composed of the 152 reflectance spectra of the Kodak Q60 target and the Munsell chips not used in the characterization process. Mean squared errors in scanner RGB values were computed as the average squared difference between the "measurements" with the actual and estimated sensitivities. These are tabulated in Table 2 for both estimation schemes, for each of the three channels. The results are significantly better than the estimated scanner SNR because of the absence of noise. However, it can be seen that the difference in the mean squared error (MSE) between POCS and PE is significantly smaller than would be expected from the mean squared estimation errors in Table 1. The primary reason for this is that the correlation matrix for

Table 2 Mean squared error in scanner RGB values obtained from the PE and POCS estimates.

\begin{tabular}{lcccc}
\hline \hline \multirow{2}{*}{ Estimation Scheme } & & \multicolumn{3}{c}{ Mean Squared Error (dB) } \\
\cline { 3 - 5 } & & Red & Green & Blue \\
\hline \multirow{2}{*}{ Illuminant unknown } & PE 2 nm & -39.12 & -47.98 & -44.79 \\
& POCS 2 nm & -28.31 & -33.24 & -43.29 \\
Iluminant known & PE 2 nm & -48.16 & -48.03 & -48.01 \\
& POCS 2 nm & -42.02 & -42.52 & -43.13 \\
& PE 10 nm & -49.81 & -47.90 & -48.90 \\
& POCS 10 nm & -43.81 & -44.22 & -39.86 \\
\hline \hline
\end{tabular}

the reflectance spectra used in the test is close to the correlation matrix of the reflectance spectra used in the PE estimation procedure.

To examine this more closely, consider the case when the illuminant is known a priori. Let $\mathbf{R}_{\text {test }}$ denote the matrix with the $K_{\text {test }}$ test reflectance spectra as its columns, and in the notation of Section 2, let $\mathbf{W}_{\text {test }}=\mathbf{L} \mathbf{R}_{\text {test }}$. The MSE in scanner RGB values is given by

$$
\frac{1}{K_{\text {test }}}\left\|\mathbf{W}_{\text {test }}^{T} \mathbf{s}-\mathbf{W}_{\text {test }}^{T} \hat{\mathbf{s}}\right\|^{2}=\frac{1}{K_{\text {test }}}(\mathbf{s}-\hat{\mathbf{s}})^{T} \mathbf{W}_{\text {test }} \mathbf{W}_{\text {test }}^{T}(\mathbf{s}-\hat{\mathbf{s}}) .
$$

The matrix ( $\left.1 / K_{\text {test }}\right) \mathbf{W}_{\text {test }} \mathbf{W}_{\text {test }}^{T}$ is the sample correlation matrix for the test spectra. In the notation of Section 3 the estimation error for the PE method can be written as

$\mathbf{s}-\hat{\mathbf{s}}=\sum_{i=P^{\prime}+1}^{N}\left(\mathbf{u}_{i}^{T} \mathbf{s}\right) \mathbf{u}_{i}-\sum_{i=1}^{P^{\prime}} \frac{1}{\sigma_{i}}\left(\mathbf{v}_{i}^{T} \boldsymbol{\epsilon}\right) \mathbf{u}_{i}$

For typical SNRs and appropriate choice of $P^{\prime}$, the first term is dominant and therefore the error in the PE estimate lies largely in the space orthogonal to that spanned by the $P^{\prime}$ principal eigenvectors of $\mathbf{W} \mathbf{W}^{T}$. Since the autocorrelation matrices $(1 / K) \mathbf{W} \mathbf{W}^{T}$ and $\left(1 / K_{\text {test }}\right) \mathbf{W}_{\text {test }} \mathbf{W}_{\text {test }}^{T}$ are close, the spaces spanned by their principal eigenvectors are also close, and therefore the error in the PE estimate is largely orthogonal to the principal eigenvectors of $\left(1 / K_{\text {test }}\right) \mathbf{W}_{\text {test }} \mathbf{W}_{\text {test }}^{T}$. Consequently the quadratic form in Eq. (21) is small.

Unfortunately, as mentioned earlier, it is a well documented fact that reflectance spectra encountered in the real world can be modeled fairly accurately using a few basis vectors. ${ }^{15-17}$ Hence the preceding problem will persist for all spectra that can be used in a physical experiment. MSE in scanner RGB values is therefore not a reliable indicator of estimation accuracy for this problem. For simulation, the problem could be circumvented by using a set of extremely narrow-band reflectances for our test "measurements," but the results in such a case would be identical to the mean squared estimation errors already tabulated in Table 1. Unfortunately, MSE in scanner RGB values is often the only criterion available for evaluating the characterization of an actual scanner where the true sensitivities are not known.

Note also that in Ref. 7 the sensitivity of a transmissive scanner was determined using smoothed least squares and minimum mean squared error estimates. However, the spectral reflectances of the calibration targets considered in the simulations here are rather smooth in comparison to the narrow-band transmission filters used in Ref. 7. Since narrow-band interferometric reflectances are expensive and sensitive to orientation, the targets considered here are more realistic and useful in a practical scenario.

\section{Experimental Results}

In this section the PE and POCS methods are compared for the characterization of an HP ScanjetIIc scanner. The scanner utilizes a fluorescent lamp whose relative spectral radi- 


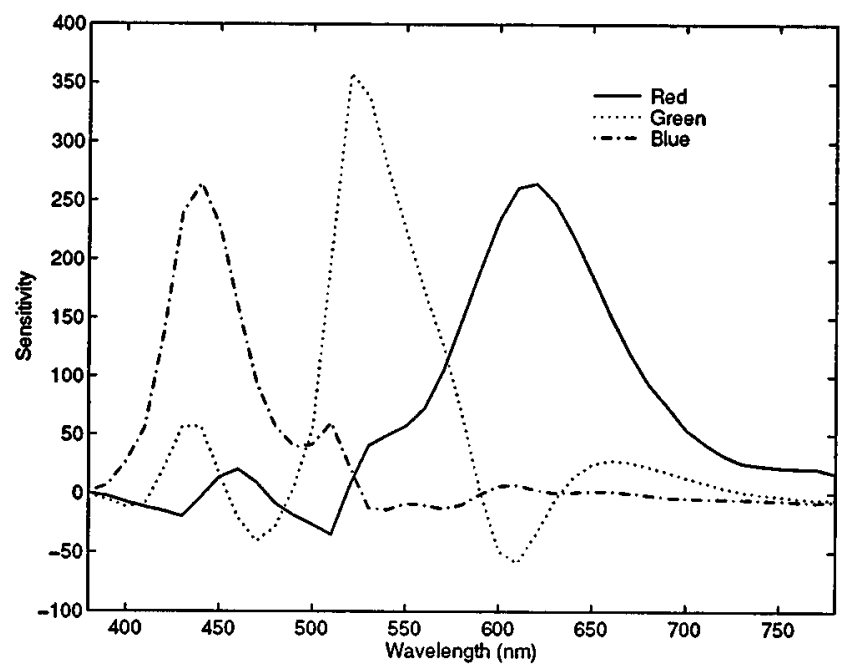

Fig. 8 PE estimates for HP Scanjetllc.

ance was measured using a spectroradiometer* and is shown in Fig. 1. For the purposes of characterization the knowledge of the scanner lamp was used for the POCS and the PE methods with a $10-\mathrm{nm}$ sampling and the illuminant model of Section 2. The Kodak Q60 target and the Munsell chips used in the simulations of the last section were scanned to obtain scanner RGB measurements. ${ }^{\dagger}$ As in the simulations, half of the scanned blocks were used as a calibration target and the other half were used as a test target. For the PE method $P=6$ was used as higher values gave rise to estimates with very large negative lobes. For the POCS method, the noise variance, outlier, nonnegativity, smoothness, and local concavity constraints were used. The estimated SNR of $33 \mathrm{~dB}$ was used along with 95 and $99 \%$ confidence levels for defining the noise variance and the outlier sets.

The estimates of the scanner sensitivity for the PE method are plotted in Fig. 8 and for the POCS method the corresponding estimates are in Fig. 9. The performance of the methods is similar to that in the simulations. The PE method yields estimates that have sharp peaks and large negative regions, while the POCS method yields estimates that satisfy constraints imposed on the sensitivities based on prior knowledge.

Since the true sensitivities for the scanner were not known, the mean squared estimation error could not be computed. As indicated in the last section, MSEs in scanner RGB values obtained using typical reflectance targets are not reliable indicators of estimation accuracy. However, the validity of the POCS estimates can be tested by comparing MSEs in scanner RGB values for a target different from the

\footnotetext{
* It was observed that the scanner lamp exhibits a warm-up time during which its spectrum undergoes a significant change. Hence, for the scanner lamp measurements and for the scans, a sufficient warm-up was allowed.

${ }^{\dagger}$ The scanner employs an internal matrixing of the data to obtain tristimulus values corresponding to the NTSC primaries. However, such a matrixing would invalidate the positivity constraint that is employed in POCS and hence raw device data was used in this work.
}

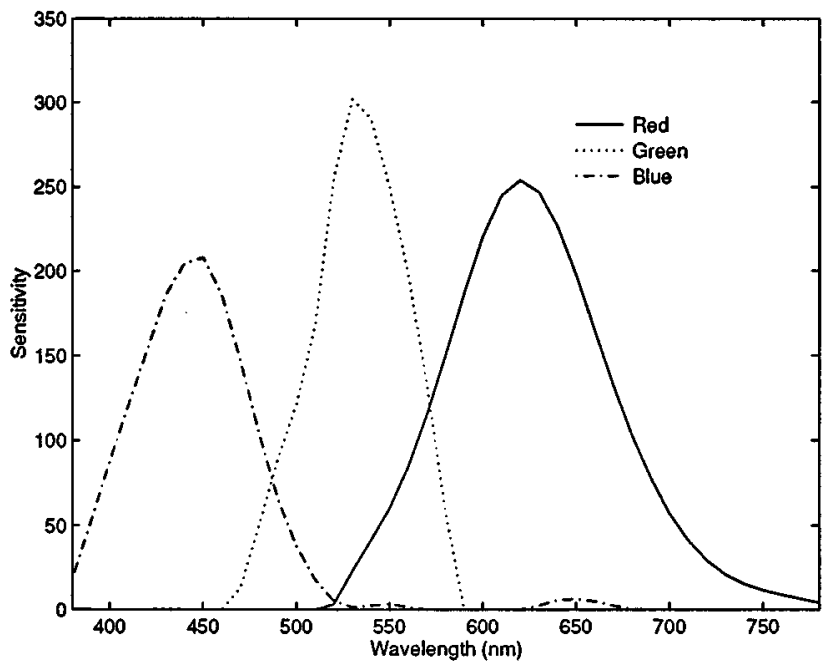

Fig. 9 POCS estimates for HP Scanjetllc.

calibration target with the imposed constraints in POCS. For this purpose, average squared differences between scanner measurements and model predictions of scanner RGB values (using estimates of scanner sensitivities) were computed for both estimation schemes over the test target. These are tabulated in Table 3. From the tabulation it can be seen that the two estimates give nearly the same performance as far as prediction of device tristimulus values is concerned. The mean squared tristimulus errors are in agreement with the estimated SNR of $33 \mathrm{~dB}$, which was used in the POCS estimation procedure.

Since MSE in scanner RGB values does not correlate well with perceptual measures of color difference, it may be argued that the error in a perceptually uniform color space such as the CIE $L^{*} a^{*} b^{*}$ space $^{36}$ is a more relevant metric for comparing the estimates. Toward this end the knowledge of scanner sensitivity may be used to determine "optimal" transformations that transform scanner RGB to the CIE $L^{*} a^{*} b^{*}$ space. ${ }^{11}$ However, such a transformation involves error not only due to the erroneous estimates of scanner sensitivity, but also due to the fact that the sensor responses are not colorimetric (do not span the human visual subspace $\left.{ }^{1}\right)$. For present commercial scanners the contribution due to the noncolorimetric nature dominates that due to the estimation errors. ${ }^{42}$ Hence, errors in the perceptually uniform space are ineffectual for comparing the performance of the estimation schemes for current desktop scanners. However, as scanners become more colorimetric the significance of a better estimate of the sensitivity will increase.

Table 3 MSE for the tristimulus values obtained from the PE and POCS estimates for HP Scanjetllc.

\begin{tabular}{lccc}
\hline \hline & \multicolumn{3}{c}{ MSE (dB) } \\
\cline { 2 - 4 } Estimate & Red & Green & Blue \\
\hline PE & -33.99 & -31.82 & -32.18 \\
POCS & -33.80 & -30.88 & -32.30 \\
\hline \hline
\end{tabular}




\section{Conclusions}

This paper investigated the use of PE and POCS estimation methods for the problem of color scanner characterization. A useful model was introduced for representing fluorescent illuminants accurately and efficiently, which greatly reduced the dimensionality of the problem. The two methods were compared through simulations and for an actual scanner characterization problem. The results indicate that when significant a priori information is available, the method of POCS outperforms the PE method, yielding significantly better estimates of the scanner sensitivities. The principal gains of the POCS method stem from the fact that it attempts to determine a smooth and continuous function determining the sensitivity as opposed to the PE scheme that estimates the samples of the sensitivity as independent parameters. The fluorescent illuminant model plays an important role in the problem by effectively increasing the available a priori information about the sensitivity.

\section{Appendix A: Model for Fluorescent Scanner Illuminant}

In this appendix, an efficient decomposition for fluorescent illuminant spectra is described and incorporated into the scanner model. The spectral sensitivities in the resulting model for the scanner's operation have a much lower dimensionality than the model based on a high spectral sampling rate.

A fluorescent lamp spectrum can be represented as the sum of a continuous spectrum emitted by the phosphors and weighted impulses corresponding to the emission lines, ${ }^{19-21}$

$l(\lambda)=c(\lambda)+\sum_{k=1}^{q} \alpha_{k} \delta\left(\lambda-\lambda_{k}\right)$

where $c(\lambda)$ is the smooth and continuous (band-limited) spectrum emitted by the phosphors, $\left\{\lambda_{1}, \lambda_{2} \ldots \lambda_{q}\right\}$ are the locations of the $q$ spectral peaks, $\alpha_{k}$ is the strength of the spectral line at wavelength $\lambda_{k}$ (emitted by the vapor in the lamp), and $\delta()$ denotes the Dirac delta function. The smooth spectrum can be represented by its samples at $10-\mathrm{nm}$ increments, thus yielding a representation of the whole spectrum in terms of these samples along with the strengths $\left\{\alpha_{k}\right\}_{k=1}^{q}$ and the locations $\left\{\lambda_{k}\right\}_{k=1}^{q}$ of the spectral peaks. Using this model greatly reduces the dimensionality of the problem compared with the uniform sampling case, and the scanner Eq. (1) now becomes

$t^{(i)}=\mathbf{r}^{T} \mathbf{L}_{c} \mathbf{m}_{i}+\sum_{k=1}^{q} \alpha_{k} m_{i}\left(\lambda_{k}\right) r\left(\lambda_{k}\right)+\epsilon^{(i)}, \quad i=1,2,3$,

where $\mathbf{L}_{c}$ is now the $N \times N$ diagonal matrix with samples of the smooth part of the illuminant spectrum, $c(\lambda)$, and the other terms are as before.

Equation (24) can be further simplified by noting that since $r(\lambda)$ and $m_{i}(\lambda)$ are band-limited, their values at the peak locations can be written as interpolated values of their samples,

$$
\begin{aligned}
& m_{i}\left(\lambda_{k}\right)=\mathbf{g}_{k}^{T} \mathbf{m}_{i} \quad k=1,2, \ldots, q, \\
& r\left(\lambda_{k}\right)=\mathbf{g}_{k}^{T} \mathbf{r} \quad k=1,2, \ldots, q,
\end{aligned}
$$

where $\mathbf{g}_{k}$ is the vector of interpolation weights that gives the value at $\lambda_{k}$ from the equispaced samples. Substitution in Eq. (24) now yields,

$$
\begin{aligned}
t^{(i)} & =\mathbf{r}^{T} \mathbf{L}_{c} \mathbf{m}_{i}+\sum_{k=1}^{q} \alpha_{k} \mathbf{m}_{i}^{T} \mathbf{g}_{k} \mathbf{g}_{k}^{T} \mathbf{r}+\boldsymbol{\epsilon}^{(i)} \\
& =\mathbf{r}^{T} \mathbf{L}_{e} \mathbf{m}_{i}+\boldsymbol{\epsilon}^{(i)}, \quad i=1,2,3
\end{aligned}
$$

where

$\mathbf{L}_{e}=\mathbf{L}_{c}+\sum_{k=1}^{q} \alpha_{k} \mathbf{g}_{k} \mathbf{g}_{k}^{T}$

is the effective illuminant matrix.

\section{Appendix B: Partial Knowledge of Illuminant Case}

This appendix demonstrates how the framework of Eq. (5) can be used when the the scanner has a fluorescent lamp and knowledge of the illuminant is limited to the location of the spectral peaks. While physically, the model of Eq. (26) of Appendix A is still valid, the effective illuminant matrix $\mathbf{L}_{e}$ in Eq. (27) is not known, and therefore cannot be used. Note, however, that Eq. (25) can be rewritten as $\mathbf{t}^{(i)}=\mathbf{W}^{T} \mathbf{s}_{i}+\boldsymbol{\epsilon}^{(i)}, \quad i=1,2,3$, where

$\mathbf{W}=\left[\begin{array}{c}\mathbf{R} \\ \mathbf{G}^{T} \mathbf{R}\end{array}\right], \quad \mathbf{s}_{i}=\left[\begin{array}{c}\mathbf{L}_{c} \mathbf{m}_{i} \\ \mathbf{D}_{\alpha} \mathbf{G}^{T} \mathbf{m}_{i}\end{array}\right]$,

where $\mathbf{G}=\left[\mathbf{g}_{1} \mathbf{g}_{2} \ldots \mathbf{g}_{q}\right]$ and $\mathbf{D}_{\alpha}$ is a diagonal matrix with $\left\{\alpha_{k}\right\}_{k=1}^{q}$ as the diagonal. Note that, as in Eq. (5), $\mathbf{W}$ represents the known information and $\left\{\mathbf{s}_{i}\right\}_{i=1}^{3}$ represent the unknown quantities to be estimated. Also observe that $\mathbf{L}_{c} \mathbf{m}_{i}$ is an $N \times 1$ vector of uniformly spaced samples of $c(\lambda) m_{i}(\lambda)$, and therefore in the set theoretic estimation scheme a smoothness constraint can be imposed on the corresponding components of $\mathbf{s}_{i}$.

\section{Appendix C: Projections onto the Constraint Sets}

The projection of $\mathbf{z} \in \mathbb{R}^{N}$ onto a convex closed set $A$ is defined as

$P_{A}(\mathbf{z})=\underset{\mathbf{y} \in \mathbf{A}}{\arg \min }\|\mathbf{y}-\mathbf{z}\|_{2}^{2}$

The projections onto $A_{n}$ and $A_{p}$ are trivial. For the other constraint sets defined above the projections for a vector outside the constraint set are summarized next.

1. Projection onto $A_{\epsilon}$ : For $\mathbf{z} \notin A_{\epsilon}$ the projection onto $A_{\epsilon}$ is calculated most readily using the SVD of $\mathbf{W}$. Using the notation of Section 3 the projection is 
$P_{A_{\epsilon}}(\mathbf{z})=\mathbf{z}+\sum_{i=1}^{P} \frac{\tau \sigma_{i}}{\left(1+\tau \sigma_{i}^{2}\right)}\left(\mathbf{v}_{i}^{t} \mathbf{e}_{0}\right) \mathbf{u}_{i}$,

where $\quad \mathbf{e}_{0}=\mathbf{t}-\mathbf{W}^{T} \mathbf{z}, \quad$ and the

Kuhn-Tucker parameter $^{38} \tau$ is the positive root of

$\sum_{i=1}^{P} \frac{\left(\mathbf{v}_{i}^{T} \mathbf{e}_{0}\right)^{2}}{\left(1+\tau \sigma_{i}^{2}\right)^{2}}+\sum_{i=P+1}^{K}\left(\mathbf{v}_{i}^{T} \mathbf{e}_{0}\right)^{2}-\nu=0$.

Since $A_{m}$ has the same form as $A_{\epsilon}$, the projection onto $A_{m}$ is obtained from the preceding equations by replacing $\mathbf{W}, P, \mathbf{t}$, and $\nu$ by $\left(\mathbf{I}-\mathbf{P}_{v}\right), 3,0$, and $\kappa$, respectively.

2. Projection onto $A_{o}^{i}$ : The projection $\mathbf{z} \notin A_{o}^{i}$ onto $A_{o}^{i}$ is given by ${ }^{43}$

$P_{A_{o}^{i}}(\mathbf{z})=\mathbf{z}+\gamma \mathbf{w}_{i}$,

where $\mathbf{w}_{i}$ is the $i$ 'th column of $\mathbf{W}$, and

$\gamma= \begin{cases}\frac{e_{0_{i}}-\xi}{\left\|\mathbf{w}_{i}\right\|^{2}}, & e_{0_{i}}>\xi \\ \frac{e_{0_{i}}+\xi}{\left\|\mathbf{w}_{i}\right\|^{2}}, & e_{0_{i}}<-\xi,\end{cases}$

where $e_{0_{i}}$ is the $i$ 'th component of $\mathbf{e}_{0}=\mathbf{t}-\mathbf{W}^{T} \mathbf{z}$.

3. Projection onto $A_{s}$ : The projection $\mathbf{x}$ of $\mathbf{z} \notin A_{s}$ onto $A_{s}$ is given by ${ }^{35,43}$

$X(k)=\frac{Z(k)}{\left(1+\tau|H(k)|^{2}\right)} \quad k=0,1, \ldots N-1$,

where $Y(k)$ denotes the discrete Fourier transform (DFT) of $\mathbf{y}$ and $\tau$ is the positive root of

$\sum_{k=0}^{N-1} \frac{|Z(k)|^{2}|H(k)|^{2}}{\left(1+\tau|H(k)|^{2}\right)^{2}}-N \mu=0$.

Once $X(k)$ is known $\mathbf{x}$ is readily found using the inverse DFT.

4. Projection onto $A_{c}^{i}$ : The projection of $\mathbf{z}$ onto $A_{c}^{i}$ is given by

$\mathbf{x}=\mathbf{z}-\frac{\mathbf{h}_{i}^{T} \mathbf{z}}{\left\|\mathbf{h}_{i}\right\|^{2}} \mathbf{h}_{i}$,

where $\mathbf{h}_{\mathbf{i}}$ is the $i$ 'th row of $\mathbf{H}$ or the (zero padded) impulse response circularly shifted $(i-1)$ times.

\section{References}

1. H. J. Trussell, "DSP solutions run the gamut for color systems,", IEEE Signal Process. Mag. 10(2), 8-23 (1993).

2. InterColor Consortium, Intercolor Profile Format, ver. 3.0 (June 1994) (available by anonymous ftp from ftp.fogra.org).

3. P. G. Engeldrum, "Color scanner colorimetric design requirements,", Proc. SPIE 1909, 75-83 (1993).

4. P. C. Hung, "Colorimetric calibration in electronic imaging devices using a look-up table model and interpolations," J. Electron. Imaging 2(1), 53-61 (1993).

5. H. R. Kang, "Color scanner calibration," J. Imaging Sci. Technol. 36(2), 162-170 (1992).

6. H. Haneishi, T. Hirao, A. Shimazu, and Y. Mikaye, "Colorimetric precision in scanner calibration using matrices," in Proc. 3rd IS\&T/
SID Color Imaging Conference: Color Science, Systems and Applications, pp. 106-108 (Nov. 1995).

7. W. K. Pratt and C. E. Mancill, "Spectral estimation techniques for the spectral calibration of a color image scanner," Appl. Opt. 15(1), 73-79 (1976).

8. J. E. Farrell and B. A. Wandell, "Scanner linearity," J. Electron. Imaging 2(3), 225-230 (1993)

9. H. J. Trussell and G. Sharma, "Signal processing methods in color calibration," in Device Independent Color Imaging, Proc. SPIE 2170, 18-23 (Feb. 1994).

10. G. Sharma and H. J. Trussell, "Characterization of scanner sensitivity," in Proc. IS\&T/SID Color Imaging Conference: Transforms and Portability of Color, pp. 103-107 (Nov. 1993).

11. M. J. Vrhel, "Mathematical methods of color correction," PhD Dissertation, North Carolina State University (May 1993).

12. R. E. Burger and D. Sherman, "Producing colorimetric data from film scanners using a spectral characterization target,' in Device Independent Color Imaging, Proc. SPIE 2170, 42-52 (1994).

13. M. A. Rodriguez and T. G. Stockham, "Producing colorimetric data from densitometric scans," Proc. SPIE 1913, 413-418 (1993).

14. P. L. Vora and H. J. Trussell, "Measure of goodness of a set of color scanning filters,", J. Opt. Soc. Am. A 10(7), 1499-1508 (1993).

15. M. J. Vrhel, R. Gershon, and L. S. Iwan, "Measurement and analysis of object reflectance spectra," Color Res. Appl. 19(1), 4-9 (1994).

16. D. Marimont and B. A. Wandell, "Linear models of surface and illuminant spectra,"' J. Opt. Soc. Am. A 9(11), 1905-1913 (1992).

17. B. A. Wandell, "The synthesis and analysis of color images," IEEE Trans. Pattern Anal. Mach. Intell. PAMI-9(1), 2-13 (1987).

18. H. J. Trussell and M. S. Kulkarni, "Sampling and processing of color signals,"' IEEE Trans. Image Process. 5, 677-681 (Apr. 1996).

19. H. J. Trussell and M. Kulkarni, "Estimation of color under fluorescent illuminants," in Proc. IEEE Intl. Conf. Image Proc. 1994, pp. III1006-1010 (1994)

20. G. Sharma and H. J. Trussell, "Decomposition of fluorescent illuminant spectra for accurate colorimetry," in Proc. IEEE Intl. Conf. Image Proc. 1994, pp. II1002-1006, (Nov. 1994).

21. D. L. MacAdam, Color Measurement: Theme and Variations, 2nd ed., Springer-Verlag, New York (1981).

22. G. Wyszecki and W. S. Stiles, Color Science: Concepts and Methods, Quantitative Data and Formulae, 2nd ed., John Wiley \& Sons, New York (1982).

23. G. W. Stewart, Introduction to Matrix Computations, Academic Press, New York (1974).

24. G. H. Golub and C. F. Van Loan, Matrix Computations, 2nd ed., The Johns Hopkins University Press, Baltimore (1989).

25. M. J. Vrhel and H. J. Trussell, "Color correction using principal components," Color Res. Appl. 17(5), 328-338 (1992).

26. R. Kumaresan and D. W. Tufts, "Estimating the parameters of exponentially damped sinusoids and pole-zero modelling in noise," IEEE Trans. Acoust. Speech Signal Process. 30(6), 833-840 (1982).

27. A. K. Jain, Fundamentals of Digital Image Processing, Prentice-Hall, Englewood Cliffs, NJ (1989).

28. P. L. Combettes, "The foundations of set theoretic estimation," Proc. IEEE 81(2), 182-208 (1993).

29. L. M. Bregman, "The method of successive projection for finding a common point of convex sets," Dokl. Akad. Nauk. USSR 162(3), 487-490 (1965).

30. L. G. Gubin, B. T. Polyak, and E. T. Raik, "The method of projections for finding the common point of convex sets," USSR Comput. Math. Phys. 7(6), 1-24 (1967).

31. A. R. De Pierro and A. N. Iusem, "A parallel projection method for finding a common point of a family of convex sets," Pesquisa $\mathrm{Op}$ eracion. 5(1), 1-20 (1985)

32. J. L. Devore, Probability and Statistics for Engineering and the Sciences, 3rd ed., Brooks/Cole Publishing, Pacific Grove, CA (1991).

33. P. L. Combettes and H. J. Trussell, "The use of noise properties in set theoretic estimation," IEEE Trans. Sig. Proc. 39(7), 1630-1641 (1991).

34. M. I. Sezan and H. J. Trussell, "Prototype image constraints for settheoretic image restoration,"' IEEE Trans. Sig. Proc. 39(10), 2275$2285(1991)$.

35. H. Stark and E. T. Olsen, "Projection-based image restoration," $J$. Opt. Soc. Am. 9(11), 1914-1919 (1992).

36. CIE, "Colorimetry," CIE publication 15.2, Technical report, Vienna (1986).

37. J. B. Cohen, "Color and color mixture: scalar and vector fundamentals," Color Res. Appl. 13(1), 5-39 (1988).

38. D. G. Luenberger, Linear and Nonlinear Programming, 2nd ed., Addison-Wesley, Reading, MA (1989).

39. H. J. Trussell and M. R. Civanlar, "The initial estimate in constrained iterative restoration," in ICASSP Proc., Vol. 2, pp. 643-1646 (Apr. 1983).

40. Schott, Optical Glass Filters, Schott Glass Technologies, Duryea, PA.

41. M. Nier and M. E. Courtot, Eds., Standards for Electronic Imaging Systems, Proc. SPIE CR37 (1991). 
42. G. Sharma and H. J. Trussell, "Color scanner performance tradeoffs," Proc. SPIE 2658, 270-278 (1996).

43. H. J. Trussell and M. R. Civanlar, "Feasible solution in signal restoration,' IEEE Trans. Acoust. Speech Signal Process. ASSP-32, 201212 (Apr. 1984).

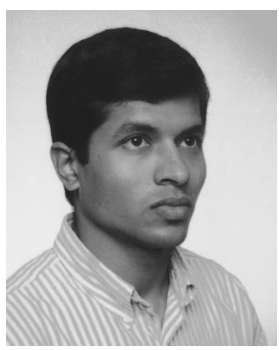

Gaurav Sharma received a BE degree in electronics and communication engineering from the University of Roorkee, India, in 1990; an ME degree in electrical communication engineering from the Indian Institute of Science, Bangalore, India, in 1992; an MS degree in applied mathematics from North Carolina State University (NCSU), Raleigh, in 1995; and a PhD in electrical engineering, also from NCSU, in 1996. From 1992 through 1996 he was a research assistant at the Center for Advanced Computing and Communications in the Electrical and Computer Engineering Department at NCSU. Since August 1996 he has been employed as a member of the research and technical staff at Xerox Corporation's Digital Imaging Technology Center in Webster, New York. His current research interests include signal restoration, color science, image halftoning, and error correction coding. Dr. Sharma is a member of Phi Kappa Phi, an associate member of Sigma Xi, and a student member of SPIE, IEEE, and the IEEE Signal Processing Society.

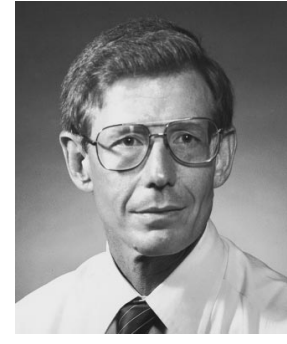

H. Joel Trussell received a BS degree from Georgia Tech in 1967, an MS degree from Florida State in 1968, and a PhD degree from the University of New Mexico in 1976. He joined the Los Alamos Scientific Laboratory, New Mexico, in 1969, where he began working in image and signal processing in 1971. During 1978 and 1979 he was a visiting professor at Heriot-Watt University, Edinburgh, Scotland, where he worked with both the university and industry on image processing problems. In 1980 he joined the Electrical and Computer Engineering Department at North Carolina State University, Raleigh. During 1988 and 1989 he was a visiting scientist at the Eastman Kodak Company in Rochester, New York. Dr. Trussell's teaching has included a wide variety of subjects in the signal and image processing areas. His research interests include estimation theory, signal and image restoration, accurate measurement and reproduction of color. He is a past associate editor for the IEEE Transactions on Acoustics, Speech, and Signal Processing and currently is an associate editor for the Signal Processing Letters. He was a member and past chairman of the Image and Multidimensional Digital Signal Processing Committee of the Signal Processing Society of the IEEE and is a fellow of the IEEE. 